\title{
Modelling of inquiry diagnosis for coronary heart disease in traditional Chinese medicine by using multi-label learning
}

\author{
Guo-Ping Liu', Guo-Zheng Li ${ }^{2 *}$, Ya-Lei Wang ${ }^{2}$, Yi-Qin Wang ${ }^{1 *}$
}

\begin{abstract}
Background: Coronary heart disease (CHD) is a common cardiovascular disease that is extremely harmful to humans. In Traditional Chinese Medicine (TCM), the diagnosis and treatment of CHD have a long history and ample experience. However, the non-standard inquiry information influences the diagnosis and treatment in TCM to a certain extent. In this paper, we study the standardization of inquiry information in the diagnosis of CHD and design a diagnostic model to provide methodological reference for the construction of quantization diagnosis for syndromes of CHD. In the diagnosis of CHD in TCM, there could be several patterns of syndromes for one patient, while the conventional single label data mining techniques could only build one model at a time. Here a novel multi-label learning (MLL) technique is explored to solve this problem.
\end{abstract}

Methods: Standardization scale on inquiry diagnosis for CHD in TCM is designed, and the inquiry diagnostic model is constructed based on collected data by the MLL techniques. In this study, one popular MLL algorithm, ML-kNN, is compared with other two MLL algorithms RankSVM and BPMLL as well as one commonly used single learning algorithm, k-nearest neighbour (kNN) algorithm. Furthermore the influence of symptom selection to the diagnostic model is investigated. After the symptoms are removed by their frequency from low to high; the diagnostic models are constructed on the remained symptom subsets.

Results: A total of 555 cases are collected for the modelling of inquiry diagnosis of CHD. The patients are diagnosed clinically by fusing inspection, pulse feeling, palpation and the standardized inquiry information. Models of six syndromes are constructed by ML-kNN, RankSVM, BPMLL and kNN, whose mean results of accuracy of diagnosis reach $77 \%, 71 \%, 75 \%$ and $74 \%$ respectively. After removing symptoms of low frequencies, the mean accuracy results of modelling by ML-kNN, RankSVM, BPMLL and kNN reach 78\%,73\%, 75\% and 76\% when 52 symptoms are remained.

Conclusions: The novel MLL techniques facilitate building standardized inquiry models in CHD diagnosis and show a practical approach to solve the problem of labelling multi-syndromes simultaneously.

\section{Background}

Coronary heart disease (CHD) is a common cardiovascular disease that is extremely harmful to humans. It is easier to find in middle and old aged people with high mortality. CHD belongs to the scope of chest heartache

\footnotetext{
* Correspondence: gzli@tongji.edu.cn; Wangyiqin2380@sina.com 'Laboratory of Information Access and Synthesis of TCM Four Diagnosis, Basic Medical College, Shanghai University of Traditional Chinese Medicine, Shanghai 201203, China

${ }^{2}$ The Key Laboratory of Embedded System and Service Computing, Ministry of Education, Department of Control Science \& Engineering, Tongji University, Shanghai 201804, China
}

(c) 2010 Liu et al; licensee BioMed Central Ltd. This is an Open Access article distributed under the terms of the Creative Commons Attribution License (http://creativecommons.org/licenses/by/2.0), which permits unrestricted use, distribution, and reproduction in any medium, provided the original work is properly cited. in Traditional Chinese Medicine (TCM); there have been extensive experiences in the diagnosis and treatment of CHD in TCM and the therapeutic effects are fairly satisfying [1]. However, TCM describes diseases by qualitative and fuzzy quantitative words: there is no clear functional relationship between the symptoms and syndromes. Currently, searching the objective and inherent relationship between the symptoms and syndromes, followed by constructing diagnostic models of syndromes is a fast developing field. Standardization and objectification of TCM diagnosis is an important and 
urgent task, which could also be fatal in scientific research, teaching and clinical practice [2].

However, there are few systematic studies of quantitative diagnosis for CHD, especially of the standardization study of inquiry diagnosis for CHD. For example, Jia et al. had investigated the contribution of symptoms to syndromes diagnosis by using a complex system focused on entropy [3]. Many authors had worked on the diagnostic standardization of many other diseases, and various techniques of multivariate statistics have been applied in the construction of diagnostic models in TCM, such as discriminant analysis and regression analysis in the diagnosis of blood stasis syndrome $[4,5]$ and stroke [6,7]. Although multivariate statistics has some superiority in the solution of quantitative diagnosis in TCM, the problem on clinical data analysis with high nonlinearity could not be solved by these techniques. Moreover, the complex interaction among different symptoms could not be reflected clearly, and the diagnostic rules of TCM could not be revealed comprehensively and widely. In this circumstance, non-linear data mining techniques are appealable in quantitative diagnosis, [8].

With the introduction of data mining techniques, investigators have applied several non-linear learning techniques into the research of diagnostic standardization and objectification in TCM, such as k nearest neighbour $(\mathrm{kNN})$, neural networks, Bayesian networks, structure equations, decision tree, genetic algorithm, etc. Some authors introduced the structure equations model into the syndrome study of chronic atrophic gastritis in $\mathrm{TCM}$, and they demonstrated that the most common symptoms of the disease in TCM are related with corresponding diagnostic indicators in accordance with clinical practice in TCM [9]. Other authors introduced Bayesian networks into the clinical analysis of blood stasis syndrome and the quantitative diagnosis with results of high accuracy [10]. An improved conjugate gradient learning algorithm was introduced into the construction of three layer forward BP network model in diabetic nephropathy with satisfied forecast results [11]. The diagnostic criterion for deficiency of yin syndrome and endothelial dysfunction symptoms by the complex system technique based on entropy was investigated, and the result demonstrated that coincidence rate was good [12].

In the above mentioned syndrome standardization and objectification studies, most of the algorithms are to solve problems of single syndrome diagnosis, i.e., single label learning. However, in clinical practice, many symptoms are presenting various syndromes. Previous studies [13] have shown that the main syndromes of CHD are deficiency accompanying with excess, e.g. deficiency of qi syndrome and blood stasis syndrome, deficiency of qi syndrome and turbid phlegm syndrome, deficiency of yang syndrome and turbid phlegm syndrome, blood stasis syndrome and Qi stagnation syndrome, as the predominant combining forms of their syndromes. But the aforementioned data mining algorithms could not forecast so many syndromes, i.e., multiple labels simultaneously. Compared with conventional learning methods, multi-label learning could identify syndrome information in TCM more effectively, and could solve the multi-label problems of one sample with several syndromes. In clinical syndrome diagnostic, the inquiry information occupies by $80 \%$. Thus the MLL technique is investigated in modelling of syndrome diagnosis of $\mathrm{CHD}$ and doing forecast in syndrome inquiry diagnosis.

In this paper, standardization scale of inquiry information is designed; 555 cases of CHD are collected following this scale, and the clinical diagnosis is performed. Based on the data set, models for multi syndromes are constructed by multi-label learning, and the influence of symptom selection on modelling is also assessed. In the Method Section, we introduce the data collecting method, kNN and the multi-label learning ML-kNN algorithms; in the Results Section, the results of models constructed based on kNN and ML-kNN is studied and the influence of symptom selection on models is assessed. In the Discussion Section, the reason why multi-label learning could improve the results is clarified. Eventually, we summarize the paper in the Conclusion Section.

\section{Methods}

\section{Data set of coronary heart disease in TCM}

In this paper, a heart system inquiry diagnosis scale [14] is designed, in which the symptoms are defined clearly, and the detailed collecting methods are listed. The scale is shown in [Additional file 1].

Inclusion criteria of the patients are: 1) The patients who meet the diagnostic criteria of CHD; 2) The patients who are informed consented.

Diagnosis criteria of the patients are in western medicine and TCM. Diagnosis criteria in western medicine are by referring to "Naming and diagnosis criteria of ischemic heart disease" issued by International Society of Cardiology and the Joint Subject Team on standardization of clinical naming in World Health Organization [15]. CHD is defined as a stenosis greater than $50 \%$ in at least one of 16 segments of the 3 major coronary arteries and their branches by coronary angiography. Diagnosis criteria in TCM are according to the "Differentiation standards for symptoms and signs of Coronary Heart Disease and Angina Pectoris in Traditional Chinese Medicine" in the "Standards for differentiation of chest pain, chest distress, palpitation, short breath or debilitation for coronary heart disease in Traditional 
Chinese Medicine" modified by China Society of Integrated Traditional Chinese and Western Medicine in 1990 and the "Guideline for Clinical study of new drugs in Chinese herbs", and the standards in textbooks $[16,17]$. After discussion with experts in cardiology, the diagnosis criteria are established.

Exclusion criteria are 1) The patients with mental diseases or with other severe diseases; 2) The patients who could not express their feeling clearly; 3) The patients who refused to participate in our study or without informed consent.

The patients with coronary heart disease are selected in Cardiology Department of Longhua Hospital Affiliated to Shanghai University of Traditional Chinese Medicine, Shuguang Hospital Affiliated to Shanghai University of Traditional Chinese Medicine, Shanghai Renji Hospital and Shanghai Hospital of Chinese Medicine. The cases with incomplete information or inconformity with the diagnosis criteria of CHD are removed. This work has been approved by the Shanghai society of medical ethics. All the patients have signed the informed consent form. Finally, a total of 555 cases are obtained in the study.

Three senior chief TCM physicians performed diagnosis individually for the 555 cases by referring to the diagnosis criteria established in the study, and the data with consistent results between 2 physicians are recorded; as for the inconsistent results, the data is not recorded until the result is consistent after discussion with the other experts.

Among the 555 patients, 265 patients are male $(47.7 \%$, with mean age of $65.15+/-13.17)$, and 290 patients are female $(52.3 \%$, with mean age of $65.24+$ /13.82). The symptoms collected for inquiry diagnosis include 8 dimensions: cold or warm, sweating, head, body, chest and abdomen, urine and stool, appetite, sleeping, mood, and gynaecology, a total of 125 symptoms. There are 15 syndromes in differentiation diagnosis, of which 6 commonly-used patterns are selected in our study, including: z1 Deficiency of heart qi syndrome; z2 Deficiency of heart yang syndrome; z3 Deficiency of heart yin syndrome; $z 4$ Qi stagnation syndrome; z5 Turbid phlegm syndrome and z6 Blood stasis syndrome.

The data set is shown in [Additional file 2].

\section{Computational methods}

In our study, we construct models of the relationship between symptoms and syndromes of inquiry diagnosis by means of the multi-label k-nearest neighbour (ML$\mathrm{kNN}$ ) algorithm. Then the results are compared with those calculated by the classical k-nearest neighbour $(\mathrm{kNN})$ algorithm.

$\mathrm{kNN}$ is an algorithm whose idea is to search for the nearest point in training data set [18]. This theory regards an instance as a point in synthesis space; thus, the label of a test instance is probably similar to those of several nearest points. Based on this theory, the algorithm of $\mathrm{kNN}$ is to search for $\mathrm{k}$ train instances nearest to the test instance, then according to their labels, to predict the label of the test instance. Compared with other mining algorithms, the advantage of $\mathrm{kNN}$ lies in simpler training process, better efficiency and forecast accuracy.

In this paper, the data set for differentiation diagnosis of CHD belongs to multi-label; whereas kNN only processes single label data sets, so the collected data set should be split into many groups of single label to be calculated. The modified algorithm is shown as below.

Step 1: Split the data set with $\mathrm{n}$ labels into $\mathrm{n}$ data sets with single label.

Step 2: Presuming there are $\mathrm{p}$ samples in the test instance, for each instance $x_{i}$, calculate the distances of $x_{i}$ to all training instances; for each instance $x_{i}$ of test data, find the $\mathrm{k}$ instances of training data with the smallest distance.

Step 3: According to the labels of $\mathrm{k}$ instances, forecast the label of each test instance $x_{i}$ (in this paper, the result is judged as positive if the number of labels is more than $\mathrm{k} / 2$, otherwise, negative). Then the forecast results of $\mathrm{p}$ test instances are obtained.

Step 4: Combine $n$ groups of results to obtain the forecast result of multiple labels and assess the forecast results according to multiple label evaluation criteria.

In clinical practice, there are many multi-label problems similar to the modelling for symptoms of inquiry diagnosis in our paper. If a single label algorithm is used, the multiple labels are usually divided and then calculated. In the multi-label data, there is much relationship among each label, so simple splitting inevitably result in data loss. For this reason, multi-label learning algorithms are developed so as to better reveal the correlation of the labels, of which multi-label kNN (ML$\mathrm{kNN}$ ) is a popular technique $[19,20]$. ML-kNN is a lazy multi-label learning algorithm developed on the basis of $\mathrm{kNN}$. Based on the theory of kNN, ML-kNN aims to find $k$ nearest instances for each test instance. In ML$\mathrm{kNN}$, the labels of test instances are judged directly by nearest instances, which is different from kNN. The algorithm is shown as below.

Step 1: Calculate the conditional probability distribution of each instance associated to each label;

Step 2: Calculate the distance between the $\mathrm{x}_{\mathrm{i}}$ test instance and the training instances; then find $\mathrm{k}$ nearest instances for $\mathrm{x}_{\mathrm{i}}$. Repeat for each test instance. 
Step 3: According to the labels of $\mathrm{k}$ training instances and the conditional probability associated to each label, forecast the probability of the $x_{i}$ instance and then acquire the forecast results (here $\geq 0.5$ is taken); Repeat for each test instance.

Step 4: Evaluate the forecast results according to multi label evaluation criteria.

Codes of both algorithms of ML-kNN and kNN are implemented on the MATLAB platform, which are shown in [Additional file 3].

To make the computational results more fruitful, the results obtained by ML-kNN and kNN are compared with those of two other multi-label learning algorithms, RankSVM [21] and BPMLL [22]. We used the default and experienced parameters values in RankSVM and BPMLL. For RankSVM, the number of hidden neurons is 8 , the max training epochs is 6. For BPMLL, the number of max iteration is 10. Parameters not mentioned were set to the default values $[21,22]$.

\section{Experimental design and evaluation}

In the CHD data set, $90 \%$ of the samples are randomized as the training set and the other $10 \%$ are as the test set. The forecast analysis of models for syndromes of inquiry diagnosis in TCM is performed after re-testing the models for 50 times and taking the mean value. Then different values of $k$ are chosen to evaluate its influence on $\mathrm{kNN}$ and ML-kNN. In this paper, $\mathrm{k}$ is chosen from $\{1,3$, $5,7,9,11\}$. According to the frequency of symptoms, the symptom features are removed as follows: the symptoms with frequencies of $\{\leq 10, \leq 20, \leq 40, \leq 70, \leq 100, \leq 150$, $\leq 200$ and $\leq 400\}$ are removed in turn, thus the symptom subsets with 150, 106, 83, 64, 52, 32 and 21 are obtained. Models of inquiry diagnosis are constructed on the basis of symptom subsets, and the influence of symptom selection on forecast model of inquiry diagnosis is investigated.

Let $X$ denote the domain of instances and let $Y=$ $\{1,2, \ldots, \mathrm{Q}\}$ be the finite set of labels. Given a training set $\mathrm{T}=\left\{\left(\mathrm{x}_{1}, \mathrm{Y}_{1}\right),\left(\mathrm{x}_{2}, \mathrm{Y}_{2}\right), \ldots,\left(\mathrm{x}_{\mathrm{m}}, \mathrm{Y}_{\mathrm{m}}\right)\right\}\left(\mathrm{x}_{\mathrm{i}} \in X, \mathrm{Y}_{\mathrm{i}} \subseteq Y\right)$, the goal of the learning system is to output a multi-label classifier h: $X \rightarrow 2^{y}$ which optimizes some specific evaluation metric. It is suppose that, given an instance $x_{i}$ and its associated label set $Y_{i}$, a successful learning system will tend to output larger values for labels in $Y_{i}$ than those not in $\mathrm{Y}_{\mathrm{i}}$, i.e. $\mathrm{f}\left(\mathrm{x}_{\mathrm{i}}, \mathrm{y}_{1}\right)>f\left(\mathrm{x}_{\mathrm{i}}, \mathrm{y}_{2}\right)$ for any $\mathrm{y}_{1} \in \mathrm{Y}_{\mathrm{i}}$ and $\mathrm{y}_{2} \notin \mathrm{Y}_{\mathrm{i}}$.

The real-valued function $\mathrm{f}(\cdot, \cdot)$ can be transformed to a ranking function $\operatorname{rank}_{\mathrm{f}}(\cdot, \cdot)$, which maps the output of $\mathrm{f}$ $\left(\mathrm{x}_{\mathrm{i}}, \mathrm{y}\right)$ for any $\mathrm{y} \in Y$ to $\{1,2, \ldots, \mathrm{Q}\}$ such that if $\mathrm{f}\left(\mathrm{x}_{\mathrm{i}}, \mathrm{y}_{1}\right)>f$ $\left(\mathrm{x}_{\mathrm{i}}, \mathrm{y}_{2}\right)$ then $\operatorname{rank}_{\mathrm{f}}\left(\mathrm{x}_{\mathrm{i}}, \mathrm{y}_{1}\right)<\operatorname{rank}_{\mathrm{f}}\left(\mathrm{x}_{\mathrm{i}}, \mathrm{y}_{2}\right)$.
To evaluate the forecast results of ML-kNN comparing with traditional $\mathrm{kNN}$, the following criteria [19] are used:

- Average_Precision evaluates the average fraction of syndrome labels ranked above a particular label which actually is in the label set as follows:

$$
\operatorname{avgprec}_{\mathrm{s}}(\mathrm{f})=\frac{1}{\mathrm{p}} \sum_{\mathrm{i}=1}^{\mathrm{p}} \frac{1}{\left|\mathrm{Y}_{i}\right|} \sum_{\mathrm{y} \in \mathrm{Y}_{\mathrm{i}}} \frac{\left|\left\{\mathrm{y}^{\prime} \mid \operatorname{rank}_{\mathrm{f}}\left(\mathrm{x}_{\mathrm{i}}, \mathrm{y}^{\prime}\right) \leq \operatorname{rank}_{\mathrm{f}}\left(\mathrm{x}_{\mathrm{i}}, \mathrm{y}\right), \mathrm{y}^{\prime} \in \mathrm{Y}_{\mathrm{i}}\right\}\right|}{\operatorname{rank}_{\mathrm{f}}\left(\mathrm{x}_{\mathrm{i}}, \mathrm{y}\right)}
$$

The performance is perfect when it is 1 ; the bigger the value of average precision, the better the performance of classifiers.

- Coverage evaluates how far we need, on the average, to go down the list of syndrome labels in order to cover all the proper labels of the instance as follows:

$$
\text { coverage }_{\mathrm{s}}(\mathrm{f})=\frac{1}{\mathrm{p}} \sum_{\mathrm{i}=1}^{\mathrm{p}} \max _{\mathrm{y} \in \mathrm{Y}_{\mathrm{i}}} \operatorname{rank}_{\mathrm{f}}\left(\mathrm{x}_{\mathrm{i}}, \mathrm{y}\right)-1
$$

It is loosely related to precision at the level of perfect recall. The smaller the value of coverage, the better the performance.

- Ranking_Loss evaluates the average fraction of syndrome label pairs that are reversely ordered for the instance as follows:

$$
\operatorname{rloss}_{\mathrm{s}}(\mathrm{f})=\frac{1}{\mathrm{p}} \sum_{\mathrm{i}=1}^{\mathrm{p}} \frac{1}{\left|\mathrm{Y}_{\mathrm{i}}\right|\left|\overline{\mathrm{Y}}_{\mathrm{i}}\right|}\left|\left\{\left(\mathrm{y}_{1}, \mathrm{y}_{2}\right) \mid \mathrm{z}\right\}\right|
$$

where $z$ means $f\left(x_{i}, y_{1}\right) \leq f\left(x_{i}, y_{2}\right),\left(y_{1}, y_{2}\right) \in Y_{i} \times\left|\bar{Y}_{i}\right|,|\bar{Y}|$ denotes the complementary set of $\mathrm{Y}$ in $Y$.

The performance is perfect when it is 0 ; the smaller the value of ranking loss, the better the performance.

\section{Results}

Forecast results of syndrome models for inquiry diagnosis On the data set with all symptoms, taken $\mathrm{k}=5$, the models of ML-kNN are built as described in the Methods Section. The mean accuracy obtained on the 6 syndrome labels is shown in Figure 1, and the results of kNN, RankSVM and BPMLL are also shown in the Figure as a comparison, where the horizontal coordinate stands for the labels of syndromes forecasted and AP means the average results of the whole labels; the longitudinal coordinate stands for forecast accuracy with $100 \%$ as the highest value. The comparative results of ML-kNN, RankSVM, BPMLL and kNN with $\mathrm{k}=5$ under three evaluation criteria are listed in Table 1.

The results in Figure 1 and Table 1 demonstrate that: 1) Comparing the forecast results of syndrome models by using ML-kNN with those by kNN, RankSVM and 


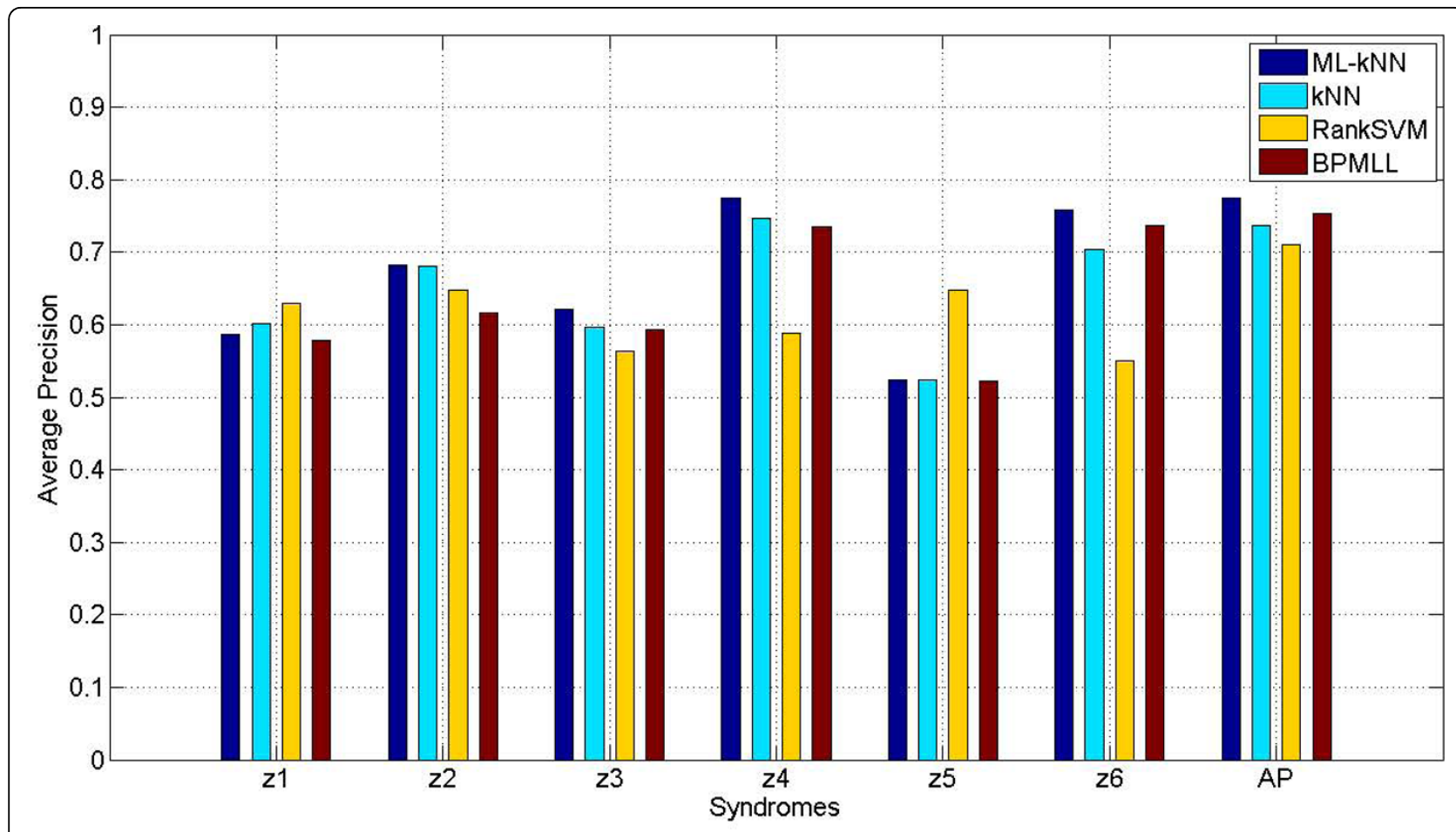

Figure 1 Results of average_precision obtained in syndrome models by using ML-kNN, RankSVM, BPMLL and kNN with $k=5$

BPMLL on the whole, the Average_Precision result of ML-kNN is $3.8 \%, 6.4 \%$ and $2.0 \%$ more than that of kNN, RankSVM and BPMLL, while the Coverage result of ML-kNN are 0.13, 0.38 and 0.05 lower than kNN, RankSVM and BPMLL, the Ranking_Loss result of ML$\mathrm{kNN}$ are $0.103,0.136$ and 0.028 lower than $\mathrm{kNN}$, RankSVM and BPMLL, respectively. According to the aforementioned evaluation criteria, the higher of Average_Precision, the better results obtained, and other measures are just the opposite. Thus the ML-kNN results are significantly better than $\mathrm{kNN}$, RankSVM and BPMLL. 2) As multi-label algorithms, BPMLL obtains better results than kNN, while RankSVM does not. 3) On each label, five out of six syndromes of ML-kNN have better forecast accuracy; the accuracy of ML-kNN is some lower than $\mathrm{kNN}$ and RankSVM only in $\mathrm{z} 1$ and z4 syndrome. 4) Different labels result in different forecast results; although clinical values are shown from the results on the whole, the result of $\mathrm{z} 5$ is slightly greater than $50 \%$.

\section{Influence on the forecast results by using different $k$} values

In order to determine whether the $\mathrm{k}$ value influenced the forecast results in ML-kNN, we construct models with $\mathrm{k}$ values as $1,3,5,7,9$ and 11 , respectively. Then the forecast results are listed in Figure 2 under the evaluation criteria of Average_Precision, Coverage and Ranking_Loss, respectively, where the horizontal coordinate stands for the $\mathrm{k}$ value, and longitudinal coordinate stands for results of Average_Precision, Coverage and Ranking_Loss, respectively.

From Figure 2, it can be seen that: 1) the forecast results of ML-kNN and kNN vary with variation of $\mathrm{k}$ values, but the change is small with a minor impact on diagnostic results with higher $k$ values, suggesting that both algorithms are stable. 2) Whatever $k$ values, the forecast Average_Precision results of ML-kNN are significantly higher than that of $\mathrm{kNN}$, while the results of Coverage and Ranking_Loss are significantly lower than $\mathrm{kNN}$, suggesting that the modelling results of ML-kNN

Table 1 Results of syndrome models for inquiry diagnosis by using ML-kNN, RankSVM, BPMLL and kNN with $k=5$

\begin{tabular}{cccc}
\hline Evaluation criteria & ML-kNN & kNN & RankSVM \\
\hline Average_Precision(\%) & $77.4 \pm 3.3$ & $73.6 \pm 3.1$ & $71.0 \pm 2.1$ \\
\hline Coverage & $3.31 \pm 0.31$ & $3.44 \pm 0.30$ & $3.69 \pm 0.28$ \\
\hline Ranking_Loss & $0.283 \pm 0.035$ & $0.386 \pm 0.037$ & $0.419 \pm 0.041$ \\
\hline
\end{tabular}



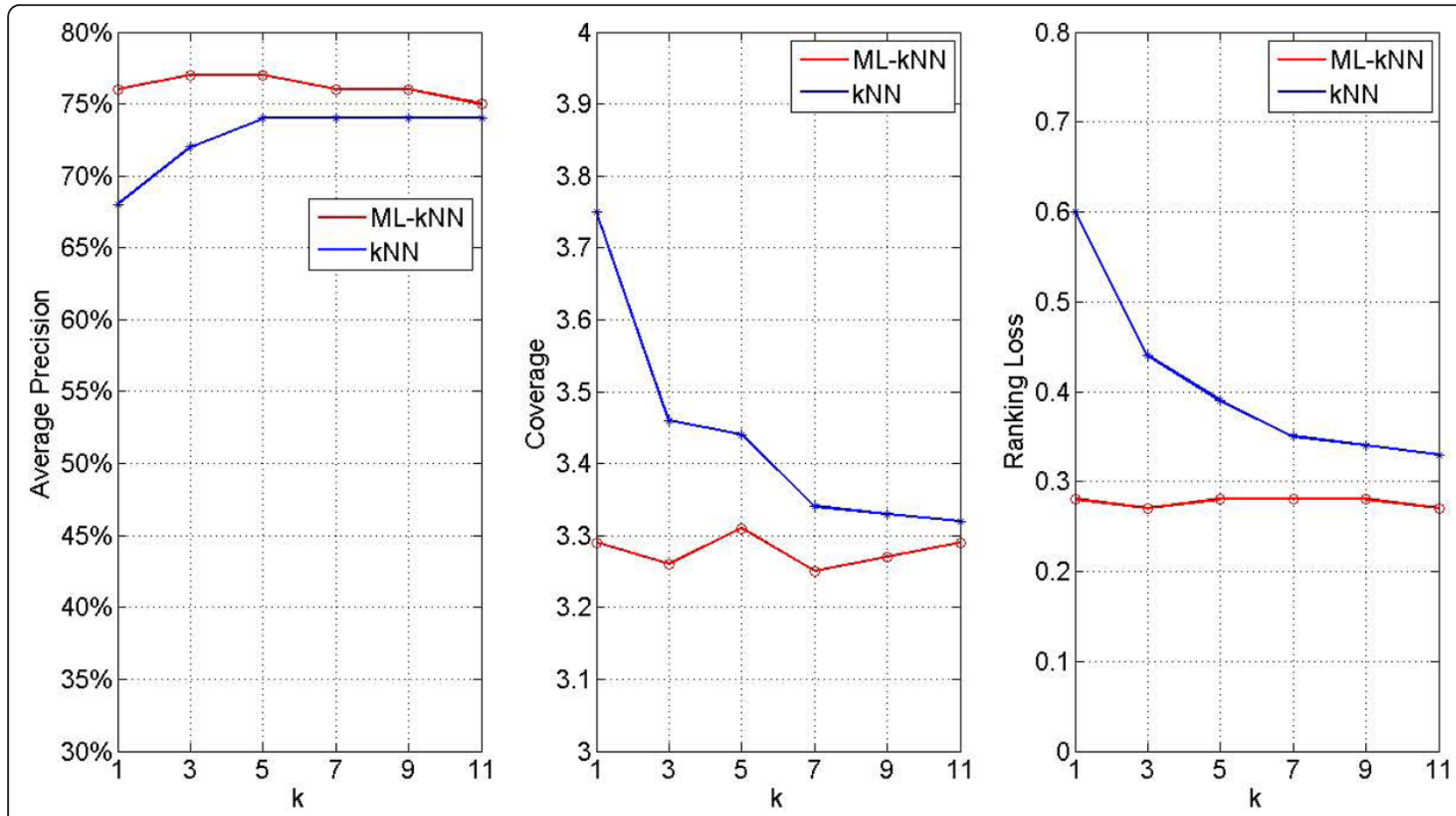

Figure 2 Results of syndrome models for inquiry diagnosis on whole labels by using ML-kNN and kNN with different $k$ values

are better than those of kNN. 3) On the criterion of Average_Precision, when taking $\mathrm{k}$ value as 5, both algorithms obtain the best forecast results. This propensity varies on the other two criteria. On the Coverage criterion, the best results are obtained when taking $\mathrm{k}$ as 7 and 9 , in ML-kNN and kNN, respectively. While on Ranking_Loss, the optimal results are obtained taking $\mathrm{k}$ as 3 and 11, respectively.

In order to further investigate the situations of each label, the forecast results of Average_Precision on different syndrome labels by using ML-kNN and kNN with different $\mathrm{k}$ values are illustrated in Figure 3, where the horizontal coordinate stands for the $\mathrm{k}$ value, and longitudinal coordinate stands for results of Average_Precision.

From Figure 3, we may find: 1) Forecast Average_Precision of ML-kNN reaches the highest mostly when $\mathrm{k}=$ 3 ; and with the increase of $\mathrm{k}$ value, the forecast accuracy decreases; 2) Forecast results of Average_Precision by using $\mathrm{kNN}$ increases with $\mathrm{k}$ value increasing, with the highest accuracy when $\mathrm{k}=7$; then it maintains steady with $\mathrm{k}$ value increasing; 3 ) As for the forecast of syndromes, some are better in ML-kNN and some are better in $\mathrm{kNN}$; on the whole, ML-kNN is better than $\mathrm{kNN}$.

\section{Influence of symptom selection on the forecast results} In our study, the symptoms in inquiry diagnosis are comprehensive, with a total of 125 symptoms. The frequency of some symptoms is high and others are low. Some symptoms influence the diagnosis of CHD to a greater extent and some to a less extent. Consequently, we select symptoms according to the frequency of symptoms, and choose the symptoms with higher frequency to investigate the influence of symptom selection on modelling.

According to the frequency of symptoms, we choose 125 symptom subset (removing those with frequency $\leq$ 10), 106 symptom subset (removing those with frequency $\leq 20$ ), 83 symptom subset (removing those with frequency $\leq 40$ ), 64 symptom subset (removing those with frequency $\leq 70$ ), 52 symptom subset (removing those with frequency $\leq 100), 32$ symptom subset (removing those with frequency $\leq 150$ ), and 21 symptom subset (removing those with frequency $\leq 200$ and $\leq$ 400). Finally, 7 subsets of symptom are obtained. On the 7 subsets, respective models are constructed by using ML-kNN, kNN, RankSVM and BPMLL, whose forecast results of three criteria are listed in Table 2.

Table 2 demonstrates that different symptom subsets leads to different forecast accuracy, but whatever the subset is, ML-kNN is superior to RankSVM, BPMLL and kNN. By using both algorithms, the forecast Average_Precision on the subset of 52 symptoms is the highest: $78.0 \%$ by $\mathrm{ML}-\mathrm{kNN}, 74.7 \%$ by $\mathrm{kNN}, 73.3 \%$ by RankSVM, and $75.1 \%$ by BPMLL. As to other two evaluation measures Coverage and Ranking_Loss, ML-kNN is also superior to RankSVM, BPMLL and kNN. 

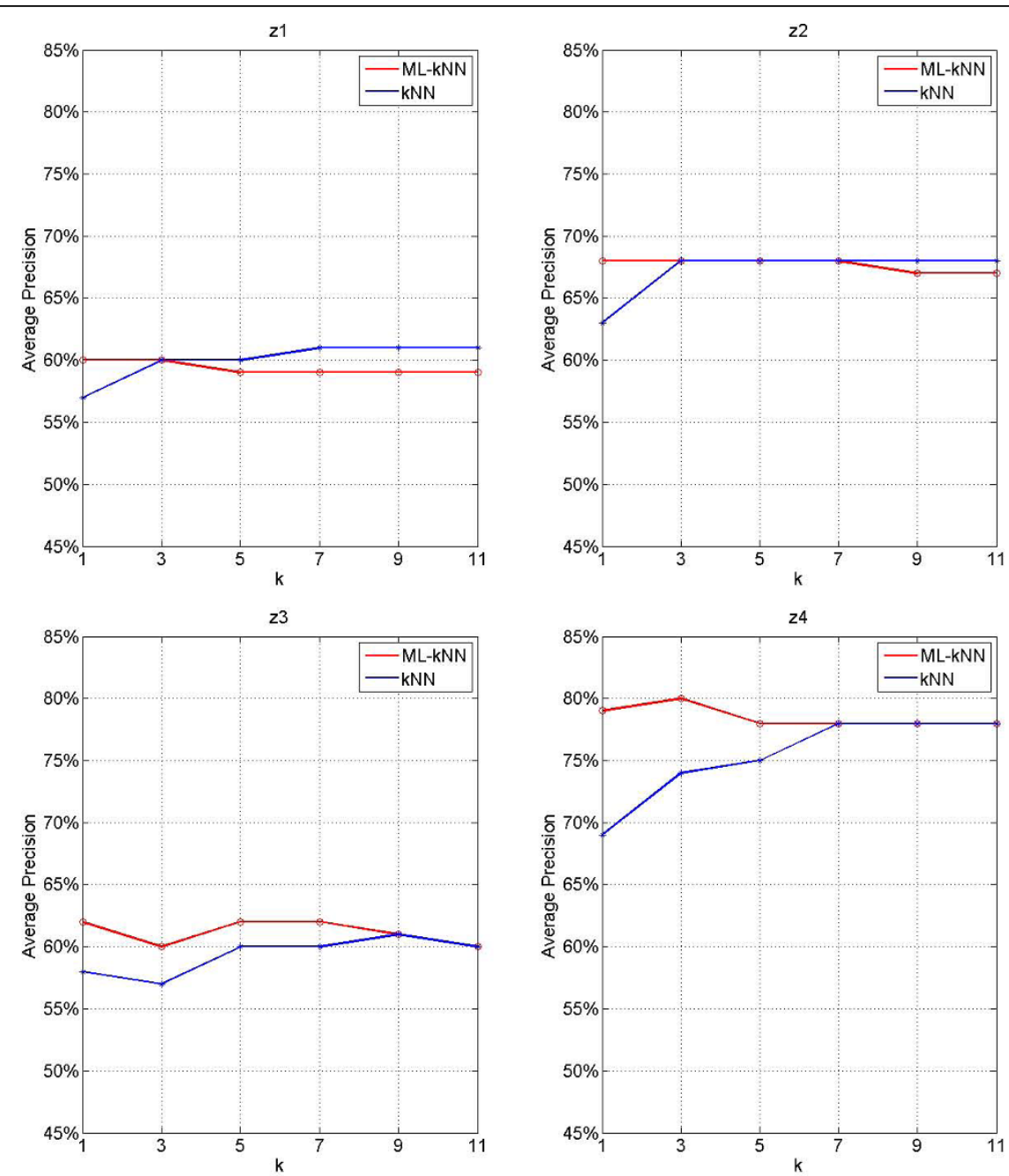

z5
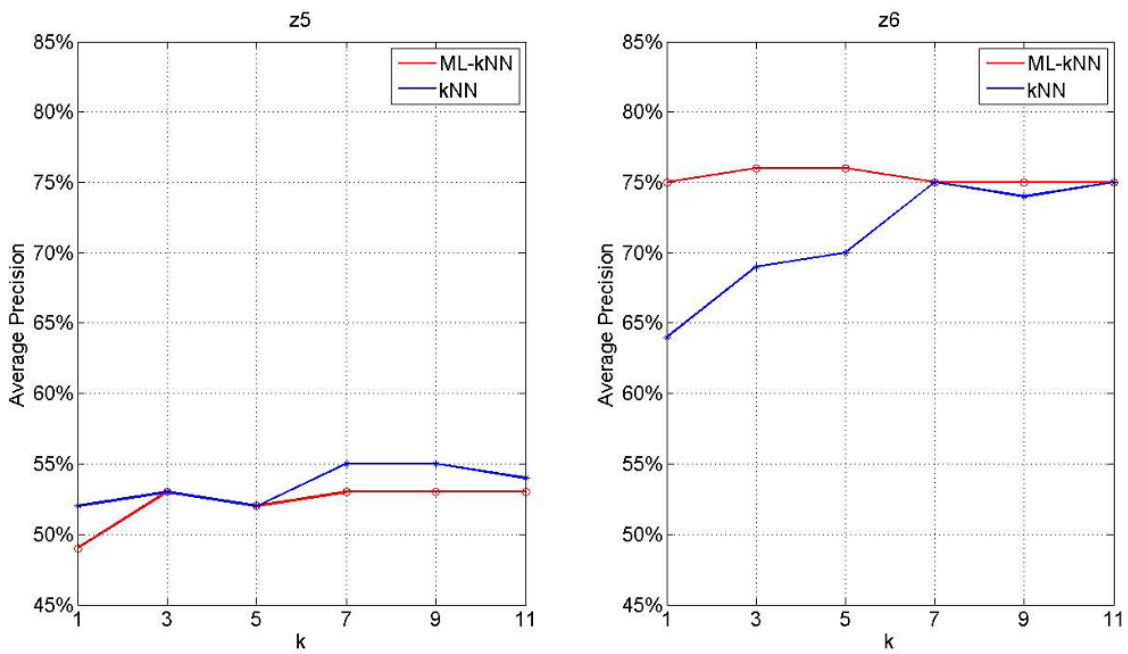

Figure 3 Results of syndrome models for inquiry diagnosis on each label by using ML-kNN and kNN with different $k$ values 
Table 2 Results of syndrome models for inquiry diagnosis on total labels by using ML-kNN, RankSVM, BPMLL and kNN with different symptom subsets

\begin{tabular}{|c|c|c|c|c|}
\hline \multirow[t]{2}{*}{ symptoms } & \multicolumn{4}{|c|}{ Average_Precision(\%) } \\
\hline & ML-kNN & kNN & RankSVM & BPMLL \\
\hline 125 & $76.2 \pm 3.1$ & $74.2 \pm 3.3$ & $70.9 \pm 3.1$ & $76.1 \pm 3.8$ \\
\hline 106 & $76.6 \pm 2.7$ & $73.7 \pm 3.3$ & $71.0 \pm 3.4$ & $75.0 \pm 3.3$ \\
\hline 83 & $76.8 \pm 2.4$ & $75.0 \pm 3.1$ & $74.3 \pm 2.9$ & $75.8 \pm 3.4$ \\
\hline 64 & $76.6 \pm 2.9$ & $75.3 \pm 2.9$ & $74.4 \pm 2.8$ & $73.9 \pm 3.9$ \\
\hline 52 & $78.0 \pm 2.4$ & $74.7 \pm 2.3$ & $73.3 \pm 2.6$ & $75.1 \pm 2.7$ \\
\hline 32 & $75.7 \pm 3.2$ & $73.7 \pm 3.5$ & $72.1 \pm 2.9$ & $75.0 \pm 2.7$ \\
\hline 21 & $74.9 \pm 2.9$ & $73.2 \pm 3.8$ & $70.5 \pm 3.5$ & $74.4 \pm 3.3$ \\
\hline \multirow[t]{2}{*}{ symptoms } & \multicolumn{4}{|c|}{ Coverage } \\
\hline & ML-kNN & kNN & RankSVM & BPMLL \\
\hline 125 & $3.28 \pm 0.32$ & $3.44 \pm 0.23$ & $3.47 \pm 0.28$ & $3.30 \pm 0.35$ \\
\hline 106 & $3.28 \pm 0.27$ & $3.41 \pm 0.31$ & $3.43 \pm 0.28$ & $3.52 \pm 0.32$ \\
\hline 83 & $3.29 \pm 0.28$ & $3.46 \pm 0.28$ & $3.38 \pm 0.29$ & $3.32 \pm 0.38$ \\
\hline 64 & $3.22 \pm 0.23$ & $3.43 \pm 0.23$ & $3.48 \pm 0.29$ & $3.41 \pm 0.28$ \\
\hline 52 & $3.21 \pm 0.24$ & $3.43 \pm 0.21$ & $3.38 \pm 0.35$ & $3.34 \pm 0.27$ \\
\hline 32 & $3.25 \pm 0.31$ & $3.49 \pm 0.35$ & $3.41 \pm 0.25$ & $3.43 \pm 0.23$ \\
\hline 21 & $3.26 \pm 0.32$ & $3.51 \pm 0.35$ & $3.53 \pm 0.36$ & $3.42 \pm 0.35$ \\
\hline \multirow[t]{2}{*}{ symptoms } & \multicolumn{4}{|c|}{ Ranking_Loss } \\
\hline & ML-kNN & kNN & RankSVM & BPMLL \\
\hline 125 & $0.290 \pm 0.031$ & $0.394 \pm 0.044$ & $0.384 \pm 0.032$ & $0.291 \pm 0.036$ \\
\hline 106 & $0.283 \pm 0.029$ & $0.390 \pm 0.037$ & $0.351 \pm 0.035$ & $0.311 \pm 0.031$ \\
\hline 83 & $0.277 \pm 0.024$ & $0.388 \pm 0.037$ & $0.329 \pm 0.031$ & $0.337 \pm 0.029$ \\
\hline 64 & $0.266 \pm 0.032$ & $0.384 \pm 0.042$ & $0.348 \pm 0.040$ & $0.330 \pm 0.027$ \\
\hline 52 & $0.271 \pm 0.028$ & $0.379 \pm 0.034$ & $0.353 \pm 0.036$ & $0.309 \pm 0.048$ \\
\hline 32 & $0.273 \pm 0.047$ & $0.402 \pm 0.036$ & $0.343 \pm 0.042$ & $0.294 \pm 0.029$ \\
\hline 21 & $0.279 \pm 0.041$ & $0.414 \pm 0.029$ & $0.369 \pm 0.044$ & $0.321 \pm 0.037$ \\
\hline
\end{tabular}

Moreover, the forecast results for each syndrome are illuminated in Figure 4, where the horizontal coordinate stands for symptom subsets, and longitudinal coordinate stands for forecast accuracy. In order to compare MLkNN, RankSVM, BPMLL and kNN accurately, the detailed results of ML-kNN, RankSVM, BPMLL and $\mathrm{kNN}$ on the optimal subset of 52 symptoms are listed in table 3.

Figure 4 shows that different symptom subsets result in different forecast accuracy; ML-kNN is rather steady without much fluctuation. Table 3 demonstrates that out of the six syndromes, four syndromes z3 Deficiency of heart yin syndrome, z4 Qi stagnation syndrome, z5 Turbid phlegm syndrome and z6 Blood stasis syndrome are better by ML-kNN than by $\mathrm{kNN}$; while their forecast accuracy in the other two syndromes z1 Deficiency of heart qi syndrome and z2 Deficiency of heart yang syndrome is slighter lower by ML-kNN than by kNN. The forecast accuracy in the $\mathrm{z} 5$ Turbid phlegm syndrome is lower by ML-kNN than by RankSVM and BPMLL. As a whole, the forecast results of ML-kNN are superior to RankSVM, BPMLL and kNN.

\section{Discussions \\ Modelling by using multi-label learning}

$\mathrm{K}$ nearest neighbour ( $\mathrm{kNN})$ is widely used in biomedical data mining field. As an extreme of minimal distance classifiers, $\mathrm{kNN}$ is simple, easy and efficient. However, when determining the label of a test sample, kNN can only do forecast once at a time, i.e., classify a syndrome to one category in TCM. But in clinical practice, there may be strong relevance among different syndromes. The syndrome of one patient mainly is the composition of several syndromes, e.g. deficiency of heart qi syndrome usually exists with qi stagnation syndrome, turbid phlegm syndrome and/or blood stasis syndrome. Due to the defects of kNN and other conventional single label mining techniques, we recommend applying novel multi-label mining algorithm ML-kNN.

Classification forecast is performed on 555 cases of inquiry diagnosis for CHD in TCM, with four algorithms, multi-label ML-kNN, RankSVM and BPMLL as well as single label kNN. Both algorithms of ML-kNN and $\mathrm{kNN}$ are compared by using different $\mathrm{k}$ values and different symptom subsets. When $\mathrm{k}=5$, the Average_Precision of ML-kNN is $77.4 \%$, significantly superior to $73.6 \%$ of $\mathrm{kNN}, 71.0 \%$ of RankSVM and $75.4 \%$ of BPMLL. On the optimal 52 symptom subset, the Average_Precision of ML-kNN is $78.0 \%$, significantly superior to $74.4 \%$ of $\mathrm{kNN}, 73.3 \%$ of RankSVM and $75.1 \%$ of BPMLL too. Similarly, the forecast accuracy of ML-kNN is obviously better than kNN, RankSVM and BPMLL.

In the $\mathrm{kNN}$ algorithm, the syndrome diagnosis of CHD is split into several single label problems to perform forecast, then the results are combined. In this process, the latent relationships among labels (syndromes) are lost. Nevertheless, in the ML-kNN algorithm, several labels (syndromes) are treated as a whole, and the relationships among labels (syndromes) are retained. Consequently, the forecast accuracy of ML-kNN is theoretically better than that of $\mathrm{kNN}$, which is also confirmed by our experiment. Meanwhile, the comparison between ML-kNN and kNN reveals the mutual relationship between symptoms in TCM. The results suggest that when performing classification forecast for data sets of inquiry diagnosis for CHD, ML-kNN achieves better results and it seems to be a practicable solution for multi-label problems in clinical diagnosis in TCM at present.

\section{The Optimal symptom subset}

The aim of symptom feature selection is to reduce dimension of the symptoms in inquiry diagnosis for $\mathrm{CHD}$, and to find the most related symptom subsets. Our results demonstrate that the forecast accuracy is 

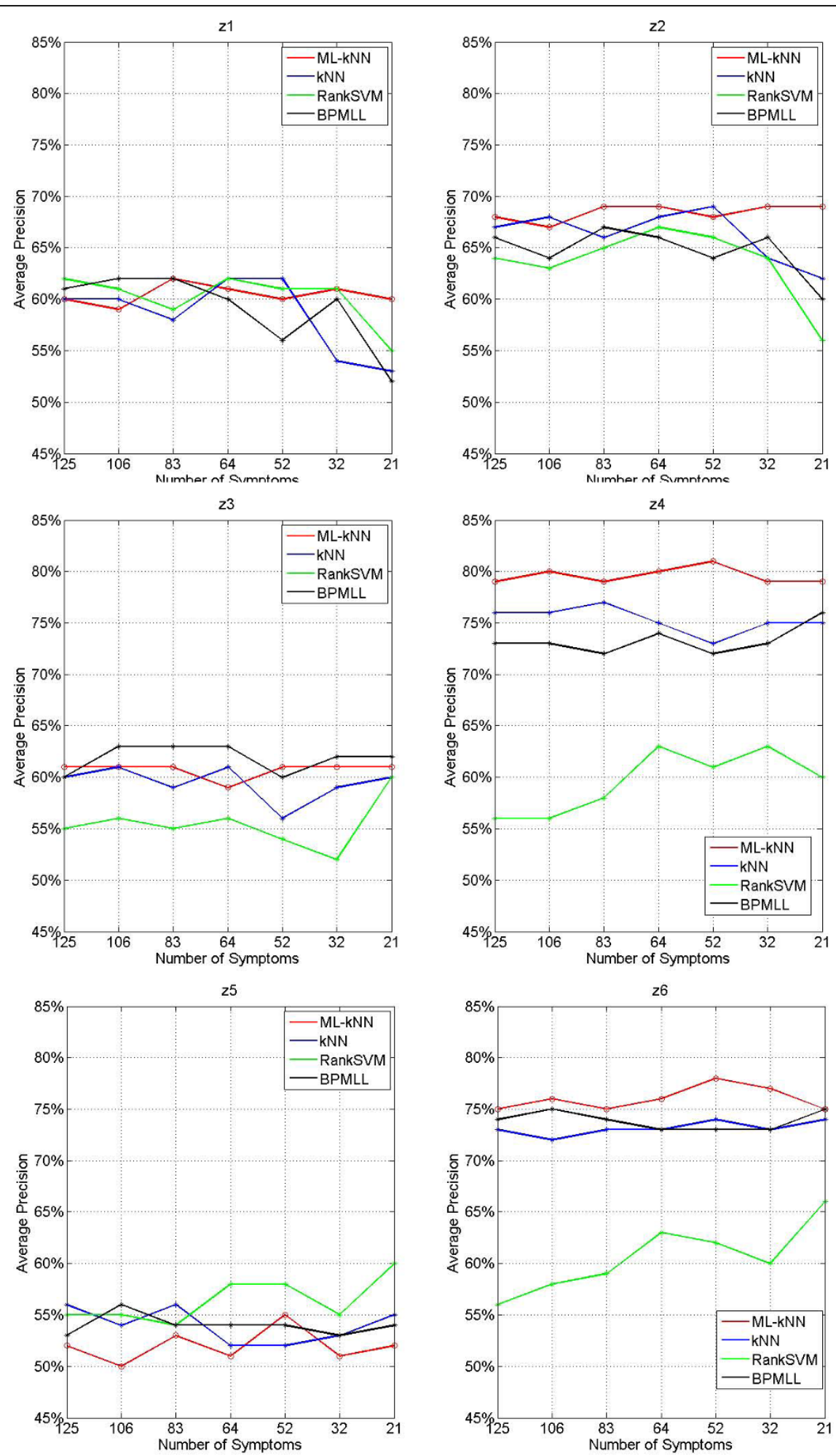

Figure 4 Results of syndrome models for inquiry diagnosis on each label by using ML-kNN, kNN, RankSVM and BPMLL with different symptom subsets 
Table 3 Results of syndrome models for inquiry diagnosis on each label by using ML-kNN, RankSVM, BPMLL and kNN on the 52-symptom subset

\begin{tabular}{ccccc}
\hline Syndromes & ML-kNN(\%) & kNN(\%) & RankSVM(\%) & BPMLL(\%) \\
\hline z1 & $60.3 \pm 2.7$ & $61.8 \pm 2.4$ & $61.1 \pm 2.9$ & $55.5 \pm 3.4$ \\
\hline z2 & $67.8 \pm 3.1$ & $68.8 \pm 3.6$ & $65.7 \pm 3.5$ & $63.5 \pm 3.4$ \\
\hline z3 & $61.1 \pm 3.3$ & $55.5 \pm 3.5$ & $54.4 \pm 3.8$ & $60.3 \pm 3.7$ \\
\hline z4 & $81.2 \pm 2.4$ & $73.4 \pm 3.2$ & $61.2 \pm 4.6$ & $71.6 \pm 2.5$ \\
\hline z5 & $54.9 \pm 1.8$ & $52.3 \pm 2.3$ & $58.0 \pm 2.4$ & $53.1 \pm 2.9$ \\
\hline z6 & $78.4 \pm 4.1$ & $73.9 \pm 4.7$ & $61.8 \pm 4.9$ & $72.7 \pm 4.3$ \\
\hline
\end{tabular}

improved after symptom selection either by ML-kNN, kNN, RankSVM or BPMLL. Furthermore, an optimized 52-symptom subset is obtained after symptom selection, shown in Table 4.

CHD belongs to the scope of heart diseases family in TCM. The main physiological functions of the heart are to control the blood vessels and govern the mind. In TCM, heart diseases result in dysfunction of blood vessels and bring on symptoms such as chest pain, choking sensation in chest, palpitation and numb of hands or feet. Dysfunction of "governing the mind" results in symptoms such as insomnia, anxiety and amnesia. In the TCM, heart is the monarch organ: it pumps blood to warm the body, and thereby it pertains to fire. Therefore, heart diseases may lead to decreased function of warm, presenting chilly or cold limb, soreness and weakness of waist and knees and night-time frequency or nocturia. Heart dominates sweat in secretion, so heart diseases may lead to selfsweating and night sweat. If the heart-fire flames up with liver-fire, it may cause irritability, impatience and bitter taste. Heart and spleen are the mother-child relationship, so heart disease may result in spleen disease and bring on the symptoms like anorexia, eating less, abdominal fullness and distension.

The duration, inducing (or aggravating) factors and relieving factors of chest pain are the main factors to determine the feature of CHD. As shown in Table 4, the above-mentioned symptoms are the main information for diagnosis of CHD. Basically, most of the information

Table 4 Frequency distribution of the optimal 52-symptom subset

\begin{tabular}{|c|c|c|c|c|c|}
\hline No. & Symptoms & Frequency & No. & Symptoms & Frequency \\
\hline 1 & X6 Duration of pain seizure & 454 & 27 & X23 Tinnitus & 195 \\
\hline 2 & X8 Relieving factor & 442 & 28 & X15 Fear of cold & 194 \\
\hline 3 & X2 Chest oppression & 436 & 29 & X28 Cough & 181 \\
\hline 4 & X5 Seizure frequency & 424 & 30 & Y52 Frequent seizure & 181 \\
\hline 5 & X4 Short breath/dyspnea/suffocation & 387 & 31 & X75 Impetuosity and susceptibility to rage & 179 \\
\hline 6 & X7 Inducing (aggravating) factor & 380 & 32 & X72 The frequent and increased urination at night & 164 \\
\hline 7 & X10 Hypodynamia & 363 & 33 & Y317 Fixed pain & 146 \\
\hline 8 & X1 Palpitation & 358 & 34 & X48 Thirst with preference for hot water & 144 \\
\hline 9 & Y31 Pain location & 348 & 35 & Y73 Aggravating gloom & 142 \\
\hline 10 & X40 Soreness and weakness of waist and knees & 282 & 36 & X29 Cough with sputum & 141 \\
\hline 11 & X3 Chest pain & 270 & 37 & X13 Amnesia & 135 \\
\hline 12 & X44 Thirsty and dry pharynx & 270 & 38 & X9 Edema & 134 \\
\hline 13 & X22 Dizziness and Blurred vision & 269 & 39 & X311 Xuli - the apex of the heart & 131 \\
\hline 14 & Y82 Relieving after administration of drug & 260 & 40 & Y731 The condition of difficult in falling asleep & 130 \\
\hline 15 & Y61 Transient & 257 & 41 & X62 Constipation & 126 \\
\hline 16 & Y72 Inducing (aggravating) after movement & 251 & 42 & X291 Color of sputum & 124 \\
\hline 17 & Y51Occasional seizure & 245 & 43 & X16 Cold limbs & 123 \\
\hline 18 & Y81 Relieving after rest & 242 & 44 & X292 Character of sputum & 120 \\
\hline 19 & X73 Insomnia & 241 & 45 & X49 Poor appetite and less amount of food & 118 \\
\hline 20 & X11 Dysphoria & 224 & 46 & X32 Gastric stuffiness & 105 \\
\hline 21 & X79 Menopause & 222 & 47 & X45 Absence of thirst and no desire for water drink & 105 \\
\hline 22 & X20 Spontaneous sweating & 217 & 48 & Y75 Inducing (aggravating) when cloudy or rainy & 103 \\
\hline 23 & X41 Numbness of hands and feet & 206 & 49 & X53 Bitter taste & 102 \\
\hline 24 & Y32 Character of pain & 205 & 50 & Y294 Difficulty or easy level of coughing with sputum & 101 \\
\hline 25 & X21 Night sweat & 201 & 51 & Y71 Seizure when quiet or without inducing factor at night & 101 \\
\hline 26 & Y62 Persistent seizure & 198 & 52 & X27 Sore-throat & 100 \\
\hline
\end{tabular}


about general pathology of CHD is listed in the table, and these items comprise the optimal symptom subset. Our results suggest that combination of symptom feature selection with classification algorithms could simplify symptom information and further improve both the comprehension and forecast accuracy of the syndromes of CHD.

Symptom feature selection by using frequency should be improved. Two cases take place out of expectation. One case is that symptoms are frequent, but they are meaningless, such as menopause. Since the average age of the females in this work is higher than 65 , so most of these females are menopause, but this has little relation with CHD. The other case is that symptoms are rare, but it is important information to diagnose the CHD, such as migratory pain. It is critical to determine the syndrome of Qi stagnation, which is removed for its low frequency. We hope to enlarge the samples and continue the study on other symptom selection methods.

\section{Conclusions}

A multi-label learning algorithm ML-kNN is employed to construct the syndrome models of inquiry diagnosis for CHD in TCM, and further produces better results than RankSVM, BPMLL and kNN do by means of three criteria of Average_Precision, Coverage and Ranking_Loss. MLkNN not only classifies the syndromes of inquiry diagnosis for CHD, but also solves the multi-label problems of one sample with several syndromes simultaneously. It overcomes the defect of conventional single label mining algorithms like $\mathrm{kNN}$ and turns out to be an effective technique for solving problems with multiple labels in clinical practice of TCM. Furthermore, combination of symptom selection with multi-label learning algorithms decreases the dimension of symptoms in inquiry diagnosis of CHD and consequently simplifies the symptom information and increases forecast accuracy. The optimal symptom subset obtained by symptom selection could also be used for guidance in clinical practice.

Future works includes designing more effective symptom selection algorithms, and employing the multi-label learning algorithms on more biomedical data sets.

\section{Additional material}

Additional file 1: The inquiry diagnostic scale of coronary heart disease in traditional Chinese medicine. Please refer to the Subsection of Data set of coronary heart disease in TCM in this paper.

Additional file 2: The collected data set of coronary heart disease in traditional Chinese medicine. Please refer to the Subsection of Data set of coronary heart disease in TCM in this paper.

Additional file 3: Source codes of ML-kNN and kNN in MATLAB language. Please refer to readme in the zip file.

\section{Acknowledgements}

This work was supported by the National Natural Science Foundation of China under Grant No.60873129 and 30901897, the Shanghai 3th Leading Academic Discipline Project Grant No. S30302, the STCSM "Innovation Action Plan" Project of China under grant no. 07DZ19726, the Shanghai Rising-Star Program under grant no. 08QA1403200 and the Open Projects Program of National Laboratory of Pattern Recognition.

\section{Author details}

'Laboratory of Information Access and Synthesis of TCM Four Diagnosis, Basic Medical College, Shanghai University of Traditional Chinese Medicine, Shanghai 201203, China. ${ }^{2}$ The Key Laboratory of Embedded System and Service Computing, Ministry of Education, Department of Control Science \& Engineering, Tongji University, Shanghai 201804, China.

\section{Authors' contributions}

All authors read and approved the final manuscript. GPL contributed to the collection, sorting, diagnostics of inquiry information of CHD and the article writing. GZL conceived and revised the paper, designed algorithms and experiments. YLW implemented the algorithms and performed the calculation. YQW contributed to the syndrome diagnosis of CHD patients.

\section{Authors' information}

Guo-Ping Liu received her Ph.D. degree from Shanghai University of Traditional TCM in 2008, and now is the assistant professor in the Fundamental Faculty of Medical College, Shanghai University of Traditional Chinese Medicine, majoring in the research of objectification of four diagnostics in traditional Chinese medicine and standardization of syndromes in traditional Chinese medicine.

Guo-Zheng Li received his Ph.D. degree from Shanghai JiaoTong University in 2004. He is currently an Associate Professor in the Department of Control Science \& Engineering, Tongji University, China. He is serving on the Committees at CCF Artificial Intelligence and Pattern Recognition Society, CAAI Machine Learning Society, International Society of Intelligent Biological Medicine and IEEE Computer Society. His research interests include feature selection, classifier design, and machine learning in bioinformatics, traditional Chinese medicine and other intelligent applications. In the recent years, $\mathrm{Li}$ has published $50+$ refereed papers in prestigious journals and conferences. He is Editors on board of IJDMB, IJMLC, IJAISC, IJFIPM, IJCBDD, JETWI, IJCIBSB and program chair of IJCBS 2009 and ITCM 2010.

Ya-Lei Wang is a M.Sc. degree student in Tongji University, majors in multilabel learning and feature selection.

Yi-Qin Wang received her Ph.D. degree from Shanghai University of Traditional Chinese Medicine in 2002, and now is the professor in the Fundamental Faculty of Medical College, Shanghai University of Traditional Chinese Medicine, doctoral degree supervisor, majoring in the research of objectification of four diagnostics in Chinese Medicine and standardization of syndromes in Chinese Medicine.

\section{Competing interests}

The authors declare that they have no competing interests.

Received: 23 March 2010 Accepted: 20 July 2010

Published: 20 July 2010

\section{References}

1. Zhang S, Song YQ, Yue W: Multicentric randomized double blinded clinical study of Yiqi Tongmai Oral Liquid against angina pectoris in patients with coronary heart disease. Chinese Journal of Integrated Traditional and Western Medicine 2007, 5(4):383-391.

2. Li JB, Zhu WF: My thinking on the disease name of TCM and its differentiation diagnosis standardization of CHD. Hunan Journal of Traditional Chinese Medicine 1994, 10(6):24.

3. Jia ZH, Li YS, Wu YL, et al: Application of entuopy-based complex systems partition method in research on quantizing TCM sydrome diagnostic criteria of angina petoris. Chinese Journal of Integrated Traditional and Western Medicine 2007, 27(9):804-806.

4. Wang J, Chen KJ: A survey on signs and symptoms about blood stasis syndrome. Liaoning Journal of Traditional Chinese Medicine 1990, 14(5):45-48. 
5. Wang J, Chen KJ, Song XH: Objectified study of abdomen diagnosis on blood stasis syndrome, Chinese. Journal of Integrated Traditional and Western Medicine 1996, 16(10):596-599.

6. Wu SX, Lin QX, Wang YY: Retrospective Study on Quantitative TCM Differential Diagnosis of Cerebral Arteriosclerosis. Journal of Beijing University of Traditional Chinese Medicine 2001, 24(1):59-63.

7. Wang Z, Zhang BL, Shen CT, et al: Multivariate Analysis of TCM Syndrome of Stroke. Chinese Journal of Integrated Traditional and Western Medicine 2003, 23(2):106-109.

8. Yuan SH, Zhang LW, Wang MQ, et al: Application of the Concept and Method of Statistics in Standardization of Syndrome Differentiation. Chinese Journal of Fundamental Medicine 2003, 9(4):15-21.

9. $L i G C$, Li $C T$, Huang $L P$, et al: An Investigation into Regularity of Syndrome Classification for Chronic Atrophic Gastritis Based on Structural Equation Model. Journal of Nanjing University of Traditional Chinese Medicine 2006, 22(4):217-220.

10. Wang XW, Qu HB, Wang J: A quantitative diagnostic method based on data-mining approach in TCM. Journal of Beijing University of Traditional Chinese Medicine 2005, 28(1):4-7.

11. Bai YJ, Meng QG, Shen HB, et al: Study on non-linear modelling of TCM syndrome of DN based on improved BP neural network model. Journal of Beijing University of Traditional Chinese Medicine 2008, 31(5):308-311.

12. Gao HL, Wu YL, Chen J, et al: Application of entropy method for complex system in study of TCM syndrome. China Journal of Traditional Chinese Medicine and Pharmacy 2008, 23(4):307-309.

13. Wang J, Xing YW, Yao KW, et al: The study on key elements of TCM syndromes and clinical application of coronary heart disease angina pectoris. Journal of Hubei University of Chinese Medicine 2009, 11(3):3-5.

14. Liu GP, Wang YQ, Dong Y, et al: Development and evaluation of Scale for heart system Inquiry of TCM. Journal of Chinese Integrative Medicine 2009, 7(1):1222-1225.

15. International Society of Cardiology and the Joint Subject Group on standardization of clinical naming in World Health Organization: Naming and diagnosis criteria of ischemic heart disease. Circulation 1979, 59(3):607.

16. Deng TT: Diagnostics of TCM. Shanghai: Shanghai Scientific and Technology Press 1984.

17. Wang YQ: Diagnostics of TCM. Beijing: Chinese Medicine Science and Technology Press 2004

18. Aha DW: Lazy learning: Special issue editorial. Artificial Intelligence Review 1997, 11(1-5):7-10

19. Zhang ML, Zhou ZH: ML-kNN: A lazy learning approach to multi-label learning. Pattern Recognition 2007, 40(7):2038-2048.

20. Tsoumakas G, Katakis I, Vlahavas I: Mining Multi-label Data. Data Mining and Knowledge Discovery Handbook Springer, 2 2009.

21. Elisseeff A, Weston J: A kernel method for multi-labelled classification. Advances in Neural Information Processing Systems MIT Press, Cambridge, MADietterich TG, Becker S, Ghahramani Z 2002, 14:681-687.

22. Zhang ML, Zhou ZH: Multilabel Neural Networks with Applications to Functional Genomics and Text Categorization. IEEE transactions on Knowledge and Data Engineering 2006, 18(10):1338-1351.

\section{Pre-publication history}

The pre-publication history for this paper can be accessed here: http://www.biomedcentral.com/1472-6882/10/37/prepub

doi:10.1186/1472-6882-10-37

Cite this article as: Liu et al:: Modelling of inquiry diagnosis for coronary heart disease in traditional Chinese medicine by using multi-label learning. BMC Complementary and Alternative Medicine 2010 10:37.

\section{Submit your next manuscript to BioMed Central and take full advantage of:}

- Convenient online submission

- Thorough peer review

- No space constraints or color figure charges

- Immediate publication on acceptance

- Inclusion in PubMed, CAS, Scopus and Google Scholar

- Research which is freely available for redistribution

Submit your manuscript at www.biomedcentral.com/submit
Biomed Central 